\title{
AUTONOMOUS EQUATIONS OF MAHLER TYPE AND TRANSCENDENCE
}

\author{
By
}

\author{
Kumiko NishioKa and Seiji NishioKa
}

\begin{abstract}
In this paper, we study transcendence of values of Mahler functions satisfying first-order rational difference equations of Mahler type with constant coefficients.
\end{abstract}

\section{Introduction and Result}

Let $K$ be an algebraic number field and $d$ an integer greater than 1. For a formal power series $f(z) \in K[[z]]$ with radius of convergence $R>0$ which satisfy the functional equation

$$
f\left(z^{d}\right)=\frac{a_{0}(z)+a_{1}(z) f(z)+\cdots+a_{m}(z) f(z)^{m}}{b_{0}(z)+b_{1}(z) f(z)+\cdots+b_{m}(z) f(z)^{m}} \quad(m \geq 1)
$$

$\mathrm{K}$. Mahler proved the following theorem, where $a_{i}(z), b_{i}(z) \in K[z]$ satisfy $a_{m}(z) \neq 0$ or $b_{m}(z) \neq 0$, and that

$$
a_{0}(z)+a_{1}(z) u+\cdots+a_{m}(z) u^{m}
$$

and

$$
b_{0}(z)+b_{1}(z) u+\cdots+b_{m}(z) u^{m}
$$

are relatively prime as polynomials in $u$. Note that at least one of these polynomials is non-constant. Let $\Delta(z)$ be their resultant.

TheOREM 1 (K. Mahler [1]). Suppose $m<d$ and that $f(z)$ is transcendental over $K(z)$. If $\alpha \in \overline{\mathbf{Q}}$ satisfies

$$
0<|\alpha|<\min \{1, R\}, \quad \Delta\left(\alpha^{d^{k}}\right) \neq 0 \quad(k \geq 0),
$$

then $f(\alpha)$ is a transcendental number.

2010 Mathematics Subject Classification: 11J91.

Key words and phrases: transcendental number, Mahler function.

Received March 4, 2015.

Revised May 7, 2015. 
However, we know few transcendental functions $f(z)$ satisfying the equation (1) in the case $m \geq 2$. In $1983, \mathrm{~K}$. Mahler obtained a necessary and sufficient condition to exist a convergent power series

$$
f(z)=f_{0}+f_{r} z^{r}\left(1+\sum_{j=1}^{\infty} \phi_{j} z^{j}\right), \quad r \geq 1, f_{r} \neq 0,
$$

satisfying a functional equation $P\left(f(z), f\left(z^{d}\right)\right)=0, P(u, v) \in K[u, v] \backslash\{0\}$, with constant coefficients. Here, we suppose that $P(u, v)$ is an irreducible polynomial with $\operatorname{deg}_{u} P=m \geq 1$ and $\operatorname{deg}_{v} P=n \geq 1$, and not a product of $u-v$ multiplied by constants. Choose an algebraic number $f_{0}$ such that $P\left(f_{0}, f_{0}\right)=0$. Thinking of the algebraic function field of one variable defined by $P(u, v)=0$, we find that there exists

$$
U_{0}(v)=f_{0}+\sum_{l=b}^{\infty} P_{l}\left(v-f_{0}\right)^{l / a}, \quad P_{b} \neq 0,1 \leq a \leq m
$$

such that $P\left(U_{0}(v), v\right)=0$, where $P_{l}(l \geq b)$ are elements of a certain finite extension $K^{\prime}$ of $K$.

TheOREM 2 (K. Mahler [2]). There exists a convergent power series

$$
f(z)=f_{0}+f_{r} z^{r}\left(1+\sum_{j=1}^{\infty} \phi_{j} z^{j}\right), \quad r \geq 1, f_{r} \neq 0,
$$

satisfying the functional equation $P\left(f(z), f\left(z^{d}\right)\right)=0$ if and only if the following three conditions hold.

(i) $b d=a$.

(ii) $r \geq 1$ and for any $l>b$ with $P_{l} \neq 0,(l d r) / a \in \mathbf{Z}$.

(iii) $f_{r}=P_{b}^{a /(a-b)}$.

Then $\phi_{j} \in K^{\prime}\left(f_{r}\right)(j \geq 1)$.

Remark. Actually, K. Mahler introduced this theorem not over $K^{\prime}$ but over C. However, we find that the proof implies the above.

We apply this theorem to the following functional equation with constant coefficients,

$$
f\left(z^{d}\right)=\frac{a_{0}+a_{1} f(z)+\cdots+a_{m} f(z)^{m}}{b_{0}+b_{1} f(z)+\cdots+b_{m} f(z)^{m}}, \quad a_{i}, b_{i} \in K, a_{m} \neq 0 \text { or } b_{m} \neq 0,
$$


where $a_{0}+a_{1} u+\cdots+a_{m} u^{m}$ and $b_{0}+b_{1} u+\cdots+b_{m} u^{m}$ are relatively prime. Let

$$
P(u, v)=v\left(b_{0}+b_{1} u+\cdots+b_{m} u^{m}\right)-\left(a_{0}+a_{1} u+\cdots+a_{m} u^{m}\right) .
$$

We suppose that $P(u, v)$ is not a product of $u-v$ multiplied by constants. We think of a solution of the form

$$
f(z)=f_{0}+f_{1} z+f_{2} z^{2}+\cdots .
$$

Since $f_{0}$ is a root of $P(u, u) \in K[u] \backslash\{0\}$, we find that $f_{0}$ is an algebraic number. We may assume $f_{0}=0$ without loss of generality, for we are only interested in the transcendence of values of $f(z)$. Then we have

$$
0=P\left(f_{0}, f_{0}\right)=P(0,0)=-a_{0},
$$

which implies $b_{0} \neq 0$. Hence we may additionally assume $b_{0}=1$. Let $s \geq 1$ be the number such that $a_{1}=\cdots=a_{s-1}=0$ and $a_{s} \neq 0$. Then we find

$$
U_{0}(v)=a_{s}^{-1 / s} v^{1 / s}+(\text { terms of higher degrees in } v),
$$

which yields $b / a=1 / s$.

We will prove that it is possible to choose $a=s$ and $b=1$. It is enough to prove

$$
P_{l} \neq 0 \Rightarrow b \mid l \text {. }
$$

Assume the contrary, and let $l_{0}=n b+k(0<k<b)$ be the minimum such that $P_{l_{0}} \neq 0$ and $b \nmid l_{0}$. By $P\left(U_{0}(v), v\right)=0$, we obtain

$$
\begin{aligned}
v(1+ & b_{1}\left(P_{b} v^{b / a}+\cdots+P_{n b} v^{n b / a}+P_{n b+k} v^{(n b+k) / a}+\cdots\right) \\
& \left.+b_{2}\left(P_{b} v^{b / a}+\cdots\right)^{2}+\cdots+b_{m}\left(P_{b} v^{b / a}+\cdots\right)^{m}\right) \\
= & a_{s}\left(P_{b} v^{b / a}+\cdots\right)^{s}+a_{s+1}\left(P_{b} v^{b / a}+\cdots\right)^{s+1}+\cdots+a_{m}\left(P_{b} v^{b / a}+\cdots\right)^{m} .
\end{aligned}
$$

For the right side, the first term whose exponent of $v^{1 / a}$ is not divisible by $b$ is

$$
a_{s} s\left(P_{b} v^{b / a}\right)^{s-1}\left(P_{n b+k} v^{(n b+k) / a}\right)=a_{s} s P_{b}^{s-1} P_{n b+k} v^{((s-1+n) b+k) / a},
$$

and for the left side, the corresponding one is

$$
v b_{1} P_{n b+k} v^{(n b+k) / a}=b_{1} P_{n b+k} v^{((s+n) b+k) / a} .
$$

Comparing the exponents, we find a contradiction. 
Hence the first condition in Theorem 2 is equivalent to $d=a=s$. Under this condition, the second condition holds for any $r \geq 1$, and so if we choose $f_{r}$ satisfying the third condition, then there exists the convergent power series $f(z) \in K^{\prime}\left(f_{r}\right)[[z]]$ such that $P\left(f(z), f\left(z^{d}\right)\right)=0$. Thus we obtain a convergent power series

$$
f(z)=f_{r} z^{r}+\cdots \in K^{\prime}\left(f_{r}\right)[[z]], \quad r \geq 1, f_{r} \neq 0,
$$

satisfying the following functional equation with constant coefficients,

$$
f\left(z^{d}\right)=\frac{a_{d} f(z)^{d}+\cdots+a_{m} f(z)^{m}}{1+b_{1} f(z)+\cdots+b_{m} f(z)^{m}},
$$

where $a_{i}, b_{i} \in K, \quad a_{m} \neq 0 \quad$ or $\quad b_{m} \neq 0, \quad a_{d} \neq 0, \quad$ and $\quad a_{d} u^{d}+\cdots+a_{m} u^{m} \quad$ and $1+b_{1} u+\cdots+b_{m} u^{m}$ are relatively prime.

Although Mahler's Theorem 1 is unsuitable for this $f(z)$ due to $d \leq m$, we have the following.

TheOREM 3 (K. Nishioka [4]). Theorem 1 still holds when $m<d^{2}$.

Theorem 1 and Theorem 3 both require transcendence of $f(z)$ over $K(z)$. Generally, it is difficult to identify transcendence of functions. However, we can use the following in this situation.

THEOREM 4 (S. Nishioka [6]). Let $f_{1}(z), \ldots, f_{n}(z) \in \mathbf{C}((z))$ satisfy the functional equations,

$$
f_{i}\left(z^{d}\right)=\frac{A_{i}\left(f_{i}(z)\right)}{B_{i}\left(f_{i}(z)\right)}, \quad i=1, \ldots, n,
$$

where $A_{i}(u), B_{i}(u) \in \mathbf{C}[u] \backslash\{0\}$ are relatively prime. If $f_{1}(z), \ldots, f_{n}(z)$ are not constants and $\max \left\{\operatorname{deg} A_{i}(u), \operatorname{deg} B_{i}(u)\right\}(i=1, \ldots, n)$ are distinct, then $f_{1}(z), \ldots, f_{n}(z)$ are algebraically independent over $\mathbf{C}$.

Since the independent variable $z$ satisfies the functional equation $f\left(z^{d}\right)=$ $f(z)^{d}$, we obtain the following as a corollary.

COROllary 5. Let $f(z) \in \mathbf{C}((z))$ be a non-constant solution of the functional equation (2). If $m \neq d$, then $f(z)$ and $z$ are algebraically independent over $\mathbf{C}$, and so $f(z)$ is transcendental over $\mathbf{C}(z)$. 
Considering all the above results, we obtain the following.

THEOREM 6. Let $d<m<d^{2}$. There exists a non-constant convergent power series $f(z) \in K^{\prime \prime}[[z]]$ satisfying the functional equation (3), where $K^{\prime \prime}$ is a certain finite extension of $K$. Let $R>0$ be the radius of convergence. If $\alpha \in \overline{\mathbf{Q}}$ satisfies

$$
0<|\alpha|<\min \{1, R\}
$$

then $f(\alpha)$ is a transcendental number.

REMARK. The condition on the resultant $\Delta(z)$ is not needed, for the equation (3) is with constant coefficients. In this case, $\Delta(z)$ is a non-zero constant.

\section{Another Example}

In this section, we study the functional equations of the form (2) with $m=d$. Note that their non-constant solutions may be algebraic over $\mathbf{C}(z)$. For example, we look at $f(z) \in \mathbf{C}[[z]] \backslash \mathbf{C}$ satisfying

$$
f\left(z^{2}\right)=\frac{f(z)^{2}}{1+c f(z)^{2}}, \quad c \in \mathbf{C} .
$$

The series $f(z)$ is related to the Mandelbrot set. It is proved that $f(z)$ is transcendental over $\mathbf{C}(z)$ if $c \neq 0$ and $c \neq-2$ in the lecture note [5] by K. Nishioka. On the other hand, $f(z)=z^{r}$ if $c=0$, and $f(z)=\left(z^{r}+z^{-r}\right)^{-1}$ if $c=-2$ (see the proof in [5]).

However, we obtain the following general result for similar functional equations.

THEOREM 7. For $d \geq 3$, a non-constant solution $f(z) \in \mathbf{C}[[z]]$ of the functional equation,

$$
f\left(z^{d}\right)=\frac{f(z)^{d}}{1+c f(z)^{d}}, \quad c \neq 0,
$$

is transcendental over $\mathbf{C}(z)$.

Proof. Assume that $f(z)$ is algebraic over $\mathbf{C}(z)$. We will derive a contradiction. By Theorem 1.3 in [5], we find $f(z) \in \mathbf{C}(z)$ (cf. Keiji Nishioka [3]). Let $g(z)=1 / f(z) \in \mathbf{C}(z)$. Then we obtain the following equation,

$$
g\left(z^{d}\right)=g(z)^{d}+c .
$$


Let

$$
g(z)=\frac{a(z)}{b(z)}
$$

where $a(z), b(z) \in \mathbf{C}[z]$ are relatively prime and $b(z)$ is monic. From the equation,

$$
\frac{a\left(z^{d}\right)}{b\left(z^{d}\right)}=\frac{a(z)^{d}}{b(z)^{d}}+c
$$

we obtain

$$
a\left(z^{d}\right) b(z)^{d}=\left(a(z)^{d}+c b(z)^{d}\right) b\left(z^{d}\right) .
$$

Since $a\left(z^{d}\right)$ and $b\left(z^{d}\right)$ are relatively prime, $b\left(z^{d}\right)$ divides $b(z)^{d}$. Comparing their degrees, we find $b\left(z^{d}\right)=b(z)^{d}$, and so $b(z)=z^{n}$. Hence

$$
g(z)=c_{1} z^{e_{1}}+\cdots+c_{t} z^{e_{t}}, \quad e_{i} \in \mathbf{Z}, e_{1}>\cdots>e_{t}, c_{1} \cdots c_{t} \neq 0 .
$$

From the above equation, we obtain

$$
c_{1} z^{d e_{1}}+\cdots+c_{t} z^{d e_{t}}=\left(c_{1} z^{e_{1}}+\cdots+c_{t} z^{e_{t}}\right)^{d}+c .
$$

In the case $t \geq 2$, the right side is

$$
\left(c_{1}^{d} z^{d e_{1}}+d c_{1}^{d-1} c_{2} z^{(d-1) e_{1}+e_{2}}+\cdots+d c_{t-1} c_{t}^{d-1} z^{e_{t-1}+(d-1) e_{t}}+c_{t}^{d} z^{d e_{t}}\right)+c .
$$

In this case, we have

$$
(d-1) e_{1}+e_{2}>(d-2) e_{1}+2 e_{2} \geq e_{1}+(d-1) e_{2} \geq e_{t-1}+(d-1) e_{t},
$$

which implies that $(d-1) e_{1}+e_{2} \neq 0$ or $e_{t-1}+(d-1) e_{t} \neq 0$, and so the right side has a term whose exponent is one of them. However, the left side of the above equation does not have such a term, for the following hold,

$$
d e_{1}>(d-1) e_{1}+e_{2}>d e_{2}
$$

and

$$
d e_{t-1}>e_{t-1}+(d-1) e_{t}>d e_{t} .
$$

Hence we conclude $t=1$, which yields

$$
c_{1} z^{d e_{1}}=c_{1}^{d} z^{d e_{1}}+c
$$

This contradicts $c \neq 0$.

By this theorem and Theorem 3, we obtain the following. 
COROLlaRY 8. Let $d \geq 3$. There exists a non-constant convergent power series $f(z) \in K^{\prime \prime}[[z]]$ satisfying

$$
f\left(z^{d}\right)=\frac{f(z)^{d}}{1+c f(z)^{d}}, \quad c \in K^{\times},
$$

where $K^{\prime \prime}$ is a certain finite extension of $K$. Let $R>0$ be the radius of convergence. If $\alpha \in \overline{\mathbf{Q}}$ satisfies

$$
0<|\alpha|<\min \{1, R\},
$$

then $f(\alpha)$ is a transcendental number.

\section{Acknowledgements}

This work was partially supported by JSPS KAKENHI Grant Number 26800049 .

\section{References}

[1] Mahler, K., Arithmetische Eigenschaften der Lösungen einer Klasse von Functionalgleichungen, Math. Ann., 101 (1929), 342-366.

[2] Mahler, K., On the analytic solution of certain functional and difference equations, Proc. Roy. Soc. London Ser. A, 389 (1983), 1-13.

[ 3 ] Nishioka, Ke., Algebraic function solutions of a certain class of functional equations, Arch. Math., 44 (1985), 330-335.

[ 4 ] Nishioka, Ku., On a problem of Mahler for transcendency of function values, J. Austral. Math. Soc. Ser. A, 33 (1982), 386-393.

[5] Nishioka, Ku., Mahler functions and transcendence, LNM Vol. 1631, Springer-Verlag, 1996.

[6] Nishioka, S., Algebraic independence of solutions of first-order rational difference equations, Results Math., 64 (2013), 423-433. doi:10.1007/s00025-013-0324-8

Kumiko Nishioka

Department of Mathematics, Hiyoshi Campus Keio University

4-1-1 Hiyoshi, Kohoku-ku, Yokohama 223-8521, Japan

Seiji Nishioka

Faculty of Science, Yamagata University

Kojirakawa-machi 1-4-12, Yamagata-shi

990-8560, Japan

E-mail: nishioka@sci.kj.yamagata-u.ac.jp 